\title{
Link Conditions for Simplifying Meshes with Embedded Structures
}

\author{
Dilip Mathew Thomas, Vijay Natarajan, Member, IEEE, and Georges-Pierre Bonneau
}

\begin{abstract}
Interactive visualization applications benefit from simplification techniques that generate good quality coarse meshes from high resolution meshes that represent the domain. These meshes often contain interesting substructures, called embedded structures, and it is desirable to preserve the topology of the embedded structures during simplification, in addition to preserving the topology of the domain. This paper describes a proof that link conditions, proposed earlier, are sufficient to ensure that edge contractions preserve topology of the embedded structures and the domain. Excluding two specific configurations, the link conditions are also shown to be necessary for topology preservation. Repeated application of edge contraction on an extended complex produces a coarser representation of the domain and the embedded structures. An extension of the quadric error metric is used to schedule edge contractions, resulting in a good quality coarse mesh that closely approximates the input domain and the embedded structures.
\end{abstract}

Index Terms-Embedded structures, extended complex, link conditions, mesh simplification, topology preservation, quadric error metric.

\section{INTRODUCTION}

$\boldsymbol{S}$ EVeral modeling and simulation applications pro$\mathcal{S}$ duce complex meshes at a very high level of detail. In order to speed up the subsequent processing, the meshes are simplified to generate a lower resolution approximation of the original mesh. A popular method used to simplify meshes is the iterative contraction of mesh edges. Many applications require that the topology of the mesh remain unchanged after simplification. In the context of edge contractions, topology preservation can be ensured by evaluating, for each edge, a set of conditions called link conditions [9] and allowing only those edges that satisfy the link conditions to be contracted.

Many meshes contain interesting substructures of lower dimensions embedded within the mesh. The topology of such embedded structures is often important. For instance, in a mesh that contains two distinct regions, the boundary separating the two regions could be an embedded structure whose topology needs to be preserved while simplifying the mesh.

Vivodtzev et al. [1], [2] have proposed a simplification technique that preserves the topology of the mesh and the embedded structures. They transform the original mesh to an extended complex by attaching simplices from a dummy vertex to simplices of the embedded

- Dilip Mathew Thomas is affiliated with the Dept. of Computer Science and Automation, Indian Institute of Science, Bangalore,

E-mail: dilip@csa.iisc.ernet.in

- Vijay Natarajan is affiliated with the Dept. of Computer Science and Automation, Supercomputer Education and Research Centre, Indian Institute of Science, Bangalore,

E-mail: vijayn@csa.iisc.ernet.in

- Georges-Pierre Bonneau is affiliated with the LJK, INRIA Grenoble, and the University of Grenoble, France,

Email: Georges-Pierre.Bonneau@inria.fr structure. Link conditions are evaluated on the extended complex and only those edges that satisfy the link conditions are contracted to simplify the mesh. They use this technique to simplify meshes with embedded structures and demonstrate, using multiple datasets, that the simplification process preserves the topology of the mesh and the embedded structures. However, the theoretical correctness of this technique is yet to be established for volumetric meshes.

The main contributions of this paper are:

- We give a mathematical proof to show that edge contractions that satisfy link conditions evaluated in the extended complex of a 3D mesh preserve the topology of the mesh and the embedded structures.

- Our proof for 3D meshes is generic. We use the same arguments to prove the analogous result for 2D meshes also.

- We extend the simplification algorithm to handle embedded structures that lie on the boundary. This was a limitation of the previous algorithm.

- We demonstrate the usefulness of the simplification technique in geophysics, isosurface topology preservation, and molecular surface topology preservation.

- We show that evaluation of link conditions on the extended complex is necessary for topology preservation of a restricted class of $2 \mathrm{D}$ meshes with $1 \mathrm{D}$ embedded structures.

Our implementation of the simplification algorithm for tetrahedral meshes uses ideas based on the Quadric Error Metric (QEM) to improve the quality of mesh elements and to approximate the scalar field defined on the mesh. Further, the algorithm also preserves the geometry of the embedded structure. Evaluation of link conditions requires computation of the order of a simplex. This 
computation is non-trivial in the context of embedded structures. We describe an explicit characterization of simplices that leads to an algorithm for computing their order.

The rest of the paper is organized as follows. Section 2 describes related work. Section 3 defines all terms used in this paper. Section 4 describes the proofs in detail. Section 5 discusses implementation of the simplification algorithm. Section 6 lists applications of the algorithm. Section 7 discusses our experimental results. Section 8 concludes the paper.

\section{Related Work}

\subsection{Mesh Simplification}

Mesh simplification is an area of active research within the scientific visualization community. Edge contractions are extensively used for mesh simplification and several algorithms exist that differ in the manner in which edges are chosen for contraction. A notable algorithm is the quadric error based algorithm of Garland et al. [6], which produces high quality approximations and is computationally very efficient. A multitude of techniques for mesh simplification are compared in several survey articles [3], [4], [5].

\subsection{Topology Preservation and Controlled Simplifi- cation}

Dey et al. [9] showed that an edge that satisfies a set of conditions, called link conditions, can be contracted without causing topology violation. These are local conditions evaluated in the neighbourhood of the edge. However, link conditions do not distinguish embedded structures from the rest of the mesh and hence may not ensure topology preservation of embedded structures.

Early work on minimum and minimal triangulations studied the smallest possible mesh that can be reached without violating the topology [15], [16]. Different from topology preserving simplification, controlled topology simplification helps to remove topological noises like small holes, while retaining important topological characteristics of the mesh. Reeb graphs and Morse-Smale complexes are extensively used for controlled topological simplification [10], [11], [12].

\subsection{Attribute Preservation}

Various attributes like material colour, scalar field, etc. are often available as attributes at each vertex of the mesh. These attributes also need to be preserved during simplification. Quadric error metric based simplification methods can be extended to enable attribute preservation [7], [8]. Cignoni et al. compared various simplification techniques to approximate the scalar field of a tetrahedral mesh [3].

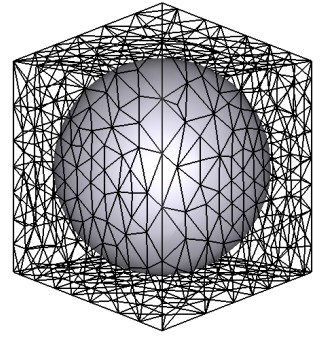

(a)

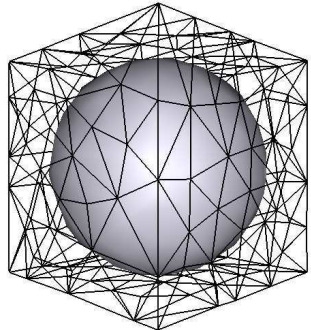

(b)
Fig. 1. (a) Original and (b) simplified volume containing embedded structure shown in grey.

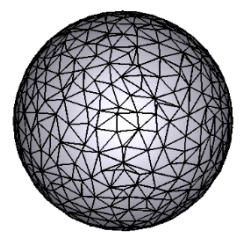

(a)

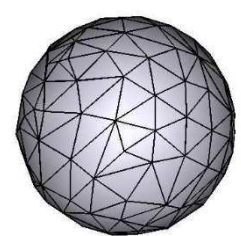

(b)
Fig. 2. (a) Embedded structure before and (b) after simplification.

\subsection{Substructure Preservation}

Mesh simplification algorithms should preserve important substructures of the mesh. We assume that the substructure is specified by the user or is available as the output of prior analysis of the mesh. Figure 1(a), 2(a) show the surface of a grey spherical ball embedded inside a cube. The mesh and the embedded structure, after simplification, is shown in Figure 1(b), 2(b).

By transforming the input mesh to an extended complex, Vivodtzev et al. [1], [2] capture the topology of the substructures in a new mesh and ensure that the topology of both the input mesh and the embedded structures is preserved during simplification.

They sketch a proof for topology preservation in the case of 2D meshes. However, this proof is not complete because their analysis overlooks the presence of new simplices in the extended complex, which are added from the dummy vertex. Moreover, the proof does not extend to the case of 3D meshes because it relies on specific properties of edges within a 2D mesh.

\subsection{Scalability}

When the size of the mesh becomes huge, out-of-core algorithms are required to process the mesh. These methods design a mesh representation scheme and a simplification algorithm that accesses the mesh in a spatially coherent manner [13], [17]. Since link conditions are evaluated in the neighbourhood of an edge, they can also be implemented out-of-core for large meshes.

\section{Definitions}

A $k$-simplex $\eta$ is the convex hull of $k+1 \geq 1$ affinely independent points. Its dimension is $\operatorname{dim}(\eta)=k$. A face $\tau$ 
of $\eta$ is the simplex defined by a non-empty subset of the $k+1$ points and $\tau$ is proper if the subset is proper. We say $\tau \leq \eta$ and call $\eta$ a coface of $\tau$. The interior of a simplex, int $\tau$ is the set of points contained in $\tau$ but not in any proper face of $\tau$. A simplicial complex $K$ is a collection of simplices such that:

i. If $\eta \in K$ then all faces of $\eta$ are also in $K$

ii. If $\eta, \tau \in K$ then $\eta \cap \tau$ is empty or a face of $\eta, \tau$.

The dimension of $K, \operatorname{dim}(K)$, is the largest dimension of simplices in $K$. The underlying space of $K,|K|$ is the union of simplex interiors in $K$. A simplex in $K$ is principal if it has no coface in $K$ other than itself. Two simplicial complexes $K$ and $L$ are said to be combinatorially equivalent, $K \simeq L$, if they have isomorphic subdivisions.

For $L \subseteq K$, the closure of $L$, denoted by $\bar{L}$ is the smallest subcomplex that contains $L$. The star of $L$ in $K$, denoted by $S t(L ; K)$, is the set of cofaces of simplices in $L$. The link of $L$ in $K$, denoted by $L k(L ; K)$, is the set of all faces of cofaces of simplices in $L$ that are disjoint from simplices in $L$.

$$
\begin{aligned}
\bar{L} & =\{\tau \in K \mid \tau \leq \eta \in L\} \\
S t(L ; K) & =\{\eta \in K \mid \eta \geq \tau \in L\} \\
L k(L ; K) & =\overline{S t(L ; K)}-S t(\bar{L} ; K)
\end{aligned}
$$

The order of a simplex $\tau$ in $K$, denoted by $\operatorname{Ord}(\tau ; K)$, measures the topological complexity of $\tau$ in $K$. Let $k=\operatorname{dim}(\operatorname{St}(\tau ; K)) \operatorname{Ord}(\tau ; K)$ is the smallest integer $i$ such that there is a $(k-i)$ simplex $\eta$, in a suitable simplicial complex $F$, such that $S t(\tau ; K)$ and $S t(\eta ; F)$ are combinatorially equivalent.

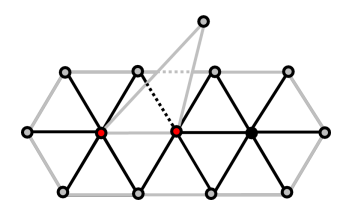

Fig. 3. The order of a simplex is an indicator of the topological complexity of its star. Grey simplices are of order 1 and the two red vertices have order 2. Other simplices have order 0 .

To illustrate order of a simplex, consider the 2D simplicial complex in Figure 3. The order of all triangles in the mesh is 0 because the star of a triangle in the mesh is the triangle itself. Hence $k=2$. Now, we can choose a triangle with its faces as a simplicial complex so that the star of the triangle is combinatorially equivalent to a triangle in the mesh. Hence $k-i=2$ and therefore $i=0$. So all triangles of the mesh have order 0 . The black edges are shared by two triangles. So $k=2$. The star of a black edge is isomorphic to a triangle subdivided into two. Thus order of black edges is also 0 . The star of the black vertex is isomorphic to subdivisions of a triangle and hence has order 0. Following a similar reasoning, all grey edges and vertices have order 1 and the two red vertices have order 2 .
The $j$-th boundary of a simplicial complex $K$, denoted by $B d_{j} K$ is the set of simplices with order greater than or equal to $j$. In Figure 3 , the $0^{t h}$ boundary is the entire mesh, the $1^{\text {st }}$ boundary is the set of grey edges, grey vertices and red vertices. The $2^{\text {nd }}$ boundary consists of the two red vertices.

For a k-simplex $\eta$ and a vertex $x$ that is affinely independent of the vertices $v_{1}, v_{2}, \ldots, v_{k+1}$ of $\eta$, the cone from $x$ to $\eta$ is defined as a simplex with vertices $x, v_{1}, v_{2}, \ldots, v_{k+1}$ and is denoted by $x \cdot \eta$.

For each $i$, define $B d_{i}^{\omega} K$ to be the simplicial complex formed from $B d_{i} K$ by adding a dummy vertex $\omega$ and adding cones from $\omega$ to all simplices in $B d_{i+1} K$. So, $B d_{i}^{\omega} K=B d_{i} K \cup\left(\omega \cdot B d_{i+1} K\right)$. For a simplex $\eta \in B d_{i}^{\omega} K$, we denote the link within $B d_{i}^{\omega} K$ as $L k_{i}^{\omega}(\eta ; K)$.

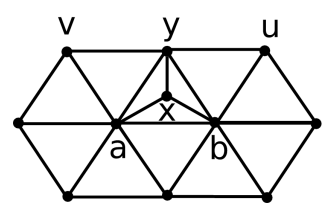

(a)

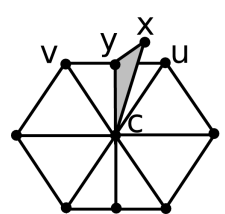

(b)
Fig. 4. (a) Simplicial complex $K$ before contracting edge $a b$. (b) After contraction, $y c$ is incident on three triangles and hence topology of $K$ is not preserved.

We use edge contraction as the basic operation for mesh simplification. While doing edge contractions in a simplicial complex $K$, topology of $K$ would be preserved if a set of conditions, called link conditions, are satisfied. For an edge $a b$, the link conditions are:

$$
L k_{i}^{\omega}(a ; K) \cap L k_{i}^{\omega}(b ; K)=L k_{i}^{\omega}(a b ; K) \quad \forall i \geq 0 .
$$

Figure 4 illustrates topology violation when an edge is contracted without checking link conditions. Edge $a b$ of a 2-complex $K$, shown in Figure 4(a), is contracted to $c$ as shown in Figure 4(b). Topology is violated since edge $y c$ after contraction is incident on three triangles, $v y c, x y c$, and uyc. However, checking link conditions prevents this situation since $x y \in L k_{0}^{\omega}(a ; K) \cap L k_{0}^{\omega}(b ; K)$ but $x y \notin L k_{0}^{\omega}(a b ; K)$.

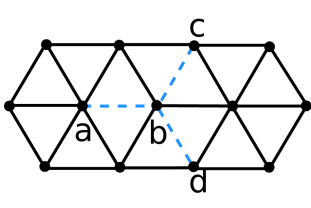

(a)

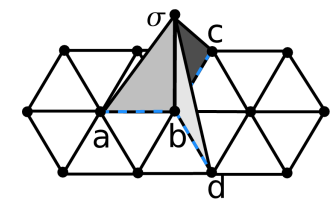

(b)
Fig. 5. (a) Simplicial complex $K$ with embedded structure in blue and (b) $\widetilde{K}$ obtained by inserting cones from $\sigma$.

For a simplicial complex $K$, an embedded structure is a user defined subcomplex of $K$ where $\operatorname{dim}(E)<\operatorname{dim}(K)$. An extended complex, $\widetilde{K}$, as defined by Vivodtzev et al. [1], [2], is obtained from $K$ by introducing a dummy vertex $\sigma$ and adding cones from $\sigma$ to simplices in $E$ so that $\widetilde{K}=K \cup \sigma \cdot E$. 
To illustrate this, consider the simplicial complex $K$ in Figure 5(a), where the edges in blue form the embedded structure. Then the extended complex is constructed by inserting cones from $\sigma$ to the blue edges as shown in Figure 5(b).

\section{Topology Preservation}

Let $K$ be a tetrahedral mesh with embedded structure $E$ of dimension 2 or lower. $\widetilde{K}$ is the extended complex formed from $K$ by adding cones to $E$ from a dummy vertex $\sigma$. Vivodtzev et al. [1], [2] assume that $K$ can be simplified without violating topology of $K$ or $E$ by contracting edges that satisfy link conditions of $\widetilde{K}$. They sketch a proof for the case $\operatorname{dim}(K)=2$. However, as indicated earlier, this proof has a major gap since it does not consider the cones added from $\sigma$ while analysing order of simplices in $\widetilde{K}$. Moreover, the proof does not extend to the case of $\operatorname{dim}(K)=3$. In this section, we present a proof for the case when $\operatorname{dim}(K)=3$. Analogous arguments prove the result for $\operatorname{dim}(K)=2$. Initially we assume that $E$ is disjoint from $B d_{1} K$ and prove that link conditions are sufficient for topology preservation of $E$ and $K$. Later, we show that this assumption can be relaxed.

Consider edge contractions of $\widetilde{K}$ where the edges are not incident on the dummy vertex $\sigma$. The link conditions for a 3-complex $\widetilde{K}$ are:

$$
\begin{aligned}
& L k_{0}^{\omega}(a ; \widetilde{K}) \cap L k_{0}^{\omega}(b ; \widetilde{K})=L k_{0}^{\omega}(a b ; \widetilde{K}), \\
& L k_{1}^{\omega}(a ; \widetilde{K}) \cap L k_{1}^{\omega}(b ; \widetilde{K})=L k_{1}^{\omega}(a b ; \widetilde{K}), \\
& L k_{2}^{\omega}(a ; \widetilde{K}) \cap L k_{2}^{\omega}(b ; \widetilde{K})=\emptyset .
\end{aligned}
$$

We want to prove that edge contractions that satisfy the above link conditions of $\widetilde{K}$ preserve topology of $K$ and $E$.

We adopt a two-step approach to prove this result. First, we show that topology of $K$ is preserved by proving that if an edge is selected for contraction then it will satisfy link conditions of $K$. This is done by proving the contrapositive statement - if an edge violates link conditions of $K$, then it will also violate link conditions of $\widetilde{K}$ and hence will not be selected for edge contraction.

Next, we show that topology of $E$ is preserved. For this, we classify edges into different categories based on whether they are part of $E$ or not. It is easy to show that edges outside $E$ that satisfy link conditions of $\widetilde{K}$ will not cause topology violation of $E$. For edges that belong to $E$, we use an approach similar to the one used for $K$ and show that link conditions of $\widetilde{K}$ are violated whenever link conditions of $E$ are violated.

\subsection{Order of a simplex in $E$ and $\widetilde{K}$}

To argue about violation of link conditions in $E$ and $\widetilde{K}$, it is important to understand the relationship between the order of a simplex in $E$ and its order in $\widetilde{K}$. The lemmas below state this relationship. For better readability, we postpone the discussion on the proof of these lemmas to Appendix A.

Lemma 1. For an edge $a b \in E$, if $\operatorname{Ord}(a b ; E)=1$, then

i. $\operatorname{Ord}(\sigma a b ; \widetilde{K})=1$

ii. $\operatorname{Ord}(a b ; \widetilde{K}) \geq 1$.

Lemma 2. For an edge $a b \in E$, if $\operatorname{Ord}(a b ; E)=0$, then $\operatorname{Ord}(\sigma a b ; \widetilde{K})=0$.

Lemma 3. For a vertex $a \in E$, if $\operatorname{Ord}(a ; E) \geq 1$, then $\operatorname{Ord}(\sigma a ; \widetilde{K}) \geq 1$.

Lemma 4. For an edge $a b \in E$, if $\operatorname{Ord}(a b ; E)=1$ and $\operatorname{Ord}(a ; E)=2$, then $\operatorname{Ord}(\sigma a ; \widetilde{K})=2$.

Lemma 5. $B d_{i}^{\omega} K \subseteq B d_{i}^{\omega} \widetilde{K}, \forall i \geq 0$.

\subsection{Preserving topology of $K$}

We now show that whenever link conditions are violated for an edge $a b$ of $K$, they are violated for $\widetilde{K}$ as well.

Let $i^{t h}$ link condition (where $i=0,1,2$ ) in $K$ be violated by a simplex $s \in B d_{i}^{\omega} K$. Note that link of an edge is contained in the link of its vertices, hence it is not possible that $s \in L k_{i}^{\omega}(a b ; K)$ and $s \notin L k_{i}^{\omega}(a ; K) \cap L k_{i}^{\omega}(b ; K)$. Hence, $s \in L k_{i}^{\omega}(a ; K) \cap L k_{i}^{\omega}(b ; K)$ and $s \notin L k_{i}^{\omega}(a b ; K)$. By Lemma $5, s \in L k_{i}^{\omega}(a ; \widetilde{K})$ and $s \in L k_{i}^{\omega}(b ; \widetilde{K})$. We show that $s \notin L k_{i}^{\omega}(a b ; \widetilde{K})$.

For a simplex $s \in E$, since $E \cap B d_{1} K=\emptyset$, $\operatorname{Ord}(s ; K)=0$. When $K$ is extended to $\widetilde{K}$, cones are added from $\sigma$ to simplices in $E$. The cones thus added to $s$ may increase the order of $s$ in $\widetilde{K}$ i.e., $\operatorname{Ord}(s ; \widetilde{K})$ may be greater than $\operatorname{Ord}(s ; K)$. Thus a new simplex that appears in $L k_{i}^{\omega}(a b ; \widetilde{K})$ is a simplex belonging to one of the following types:

I. a cone from $\sigma$

II. a cone from $\omega$ to simplices incident on $\sigma$

III. a cone from $\omega$ to simplices in $E$

IV. the subcomplex $E$ (if $i>0$ ).

If any of these new simplices in $L k_{i}^{\omega}(a b ; \widetilde{K})$ is $s$, link conditions in $\widetilde{K}$ would be satisfied. However, we show that none of the new simplices lie in $B d_{i}^{\omega} K$ and hence cannot be $s$ since $s \in B d_{i}^{\omega} K$. Type I or type II is not in $B d_{i}^{\omega} K$ since the dummy vertex $\sigma \notin B d_{i}^{\omega} K$. Type III is not in $B d_{i}^{\omega} K$ because in $B d_{i}^{\omega} K$, cones from $\omega$ are added only to simplices whose order is higher than 0 . However, all simplices in $E$ have order 0 in $K$. Type IV is not in $B d_{i}^{\omega} K$ because all simplices in $E$ have order 0 and hence do not belong to $B d_{i}^{\omega} K, i>0$. Hence, none of the new simplices in $L k_{i}^{\omega}(a b ; \widetilde{K})$ can be $s$.

Thus the simplex $s$ lies in both $L k_{i}^{\omega}(a ; \widetilde{K})$ and $L k_{i}^{\omega}(b ; \widetilde{K})$ but does not lie in $L k_{i}^{\omega}(a b ; \widetilde{K})$. So $i^{t h}$ link condition of $\widetilde{K}$ is violated.

\subsection{Preserving topology of $E$}

An edge $a b$ either belongs to the subcomplex $E$ or lies outside $E$. In either case we show that if contracting $a b$ does not violate link conditions in $\widetilde{K}$ then the topology of $E$ is preserved. 


\subsubsection{Contracting edges not in $E$}

Let $a b$ denote an edge in $\widetilde{K}$ that is not contained in $E$. Let $c$ be the new vertex obtained after contracting $a b$. Let $F$ denote the embedded structure after contracting $a b$. A simplex $\left\langle v_{1}, \ldots, v_{n}, c\right\rangle$ lies in $F$ if and only if either $<v_{1}, \ldots, v_{n}, a>$ or $\left\langle v_{1}, \ldots, v_{n}, b>\right.$ lies in $E$.

Case 1. $a, b \notin E$ :

None of the vertices of $E$ are affected by the contraction. So $F=E$ and topology of $E$ is preserved.

Case 2. $a \in E ; b \notin E$ :

After contracting $a b$, all simplices $<v_{1}, \ldots, v_{n}, a>\in E$ become $\left\langle v_{1}, \ldots, v_{n}, c>\in F\right.$. This renaming of a vertex does not change the topology of $E$.

Case 3. $a, b \in E ; a b \notin E$ :

This edge contraction is rejected because link condition (III.0) is violated. Cones are added from $\sigma$ to $a$ and $b$ but not to $a b$. So, $\sigma \in L k_{0}^{\omega}(a ; \widetilde{K}) \cap L k_{0}^{\omega}(b ; \widetilde{K})$ but $\sigma \notin L k_{0}^{\omega}(a b ; \widetilde{K})$.

\subsubsection{Contracting edges in $E$}

The embedded structure $E$ is a 2-complex. The two link conditions corresponding to $B d_{0}^{\omega} E$ and $B d_{1}^{\omega} E$ are:

$$
\begin{aligned}
& L k_{0}^{\omega}(a ; E) \cap L k_{0}^{\omega}(b ; E)=L k_{0}^{\omega}(a b ; E), \\
& L k_{1}^{\omega}(a ; E) \cap L k_{1}^{\omega}(b ; E)=\emptyset .
\end{aligned}
$$

We show that if the $i^{\text {th }}$ link condition, $(i=0,1)$, is violated in $E$, then $i^{\text {th }}$ link condition in $\widetilde{K}$ is also violated. First, we show that if $a b \in B d_{i} E$, then $a b \in B d_{i} \widetilde{K}$, so that it is meaningful to talk about contracting $a b$ in $i^{\text {th }}$ boundary of $\widetilde{K}$.

It is clear that $a b \in B d_{0} \widetilde{K}$ since $a b \in E \subset K \subset \widetilde{K}$. Now, suppose $a b \in B d_{1} E$. The order of an edge in a 2-complex cannot be two or higher. So, $\operatorname{Ord}(a b ; E)=1$. Using Lemma 1, $a b \in B d_{1} \widetilde{K}$. Thus, if $a b \in B d_{i} E$, then $a b \in B d_{i} \widetilde{K}$.

Now, suppose $i^{\text {th }}$ link condition is violated in $E$ while contracting edge $a b$. Then there is a simplex $s \in B d_{i}^{\omega} E$ such that $s \in L k_{i}^{\omega}(a ; E), s \in L k_{i}^{\omega}(b ; E)$ and $s \notin L k_{i}^{\omega}(a b ; E)$.

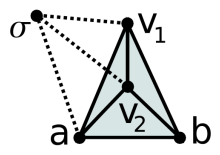

(a)

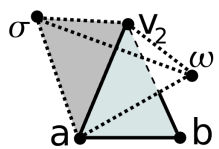

(b)

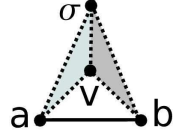

(c)

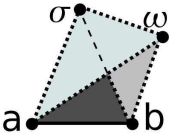

(d)
Fig. 6. Different cases in which link conditions are violated. Link of a contains (a) $\sigma v_{1} v_{2}$ (b) $\omega \sigma v_{2}$ (c) $\sigma v$ (d) $\omega \sigma$.

Case 1. Link condition (II.0) is violated: Since $B d_{0}^{\omega} E$ is a 2-complex, $s$ is either an edge or a vertex.

Case 1a. $s$ is an edge, say $v_{1} v_{2}$ : If $v_{1} \neq \omega$ and $v_{2} \neq \omega$, then $a v_{1} v_{2} \in E$ and hence $\sigma a v_{1} v_{2} \in \widetilde{K}$, see Figure 6(a). So, $\sigma v_{1} v_{2} \in L k_{0}^{\omega}(a ; \widetilde{K})$. Similarly, $\sigma v_{1} v_{2} \in L k_{0}^{\omega}(b ; \widetilde{K})$. Therefore, $L k_{0}^{\omega}(a ; \widetilde{K}) \cap$ $L k_{0}^{\omega}(b ; \widetilde{K})$ contains a triangle.

Now, assume that either $v_{1}$ or $v_{2}$ is the dummy vertex $\omega$. Without loss of generality, let $v_{1}$ be the dummy vertex. Since cones from $\omega$ are added only to simplices in $B d_{1} E, \operatorname{Ord}\left(a v_{2} ; E\right)=1$. Using Lemma 1, $\operatorname{Ord}\left(\sigma a v_{2} ; \widetilde{K}\right)=1$ and so $\omega \sigma a v_{2} \in B d_{0}^{\omega} \widetilde{K}$, see Figure 6(b). Hence $\omega \sigma v_{2} \in L k_{0}^{\omega}(a ; \widetilde{K})$. Similarly, $\omega \sigma v_{2} \in L k_{0}^{\omega}(b ; \widetilde{K})$. Therefore, $L k_{0}^{\omega}(a ; \widetilde{K}) \cap L k_{0}^{\omega}(b ; \widetilde{K})$ contains a triangle. In both cases the link condition (III.0) is violated because $L k_{0}^{\omega}(a b ; \widetilde{K})$ cannot contain a triangle.

Case 1b. $s$ is a vertex, say $v$ :

If $v \neq \omega$, then $a v, b v \in E$ and $a b v \notin E$ which implies $\sigma a v, \sigma b v \in B d_{0}^{\omega} \widetilde{K}$ and $\sigma a b v \notin B d_{0}^{\omega} \widetilde{K}$. Hence $\sigma v \in L k_{0}^{\omega}(a ; \widetilde{K}) \cap$ $L k_{0}^{\omega}(b ; \widetilde{K})$ and not in $L k_{0}^{\omega}(a b ; \widetilde{K})$, see Figure $6(\mathrm{c})$. Thus link condition (III.0) is violated.

If $v$ is $\omega$, then since link condition (II.0) is violated, $\omega \notin L k_{0}^{\omega}(a b ; E)$. This implies $\omega a b \notin B d_{0}^{\omega} E$, and therefore $\operatorname{Ord}(a b ; E)=0$. Since $\omega \in L_{0}^{\omega}(a ; E)$, it follows that $\operatorname{Ord}(a ; E) \geq 1$, because cones from $\omega$ are added only to simplices in $B d_{1} E$. Using Lemma 3, $\operatorname{Ord}(\sigma a ; \widetilde{K}) \geq 1$. Hence $\omega \sigma a \in B d_{0}^{\omega} \widetilde{K}$ and $\omega \sigma \in L k_{0}^{\omega}(a ; \widetilde{K})$, see Figure 6(d). Similarly, $\omega \sigma \in L k_{0}^{\omega}(b ; \widetilde{K})$. However, since $\operatorname{Ord}(a b ; E)=0$, it follows from Lemma 2, that $\operatorname{Ord}(\sigma a b ; \widetilde{K})=0$ and hence $\omega \sigma \notin L k_{0}^{\omega}(a b ; \widetilde{K})$. Therefore link condition (III.0) is violated.

Case 2. Link condition (II.1) is violated:

Since $B d_{1}^{\omega} E$ is a 1-complex, $s$ is a vertex, say $v$.

Case 2a. $v \in E$ :

$\operatorname{Ord}(a v ; E)=1$ since $B d_{1}^{\omega} E$ is a 1-complex.

Using Lemma 1, $\operatorname{Ord}(\sigma a v ; \widetilde{K})=1$, which implies $\sigma v \in L k_{1}^{\omega}(a ; \widetilde{K})$, see Figure 6(c). Similarly, $\sigma v \in L k_{1}^{\omega}(b ; \widetilde{K})$. Therefore, $L k_{1}^{\omega}(a ; \widetilde{K}) \cap L k_{1}^{\omega}(b ; \widetilde{K})$ contains an edge.

Case 2b. $v$ is $\omega$ :

Since in $B d_{1}^{\omega} E$, cones from $\omega$ are added only to vertices of order $2, \operatorname{Ord}(a ; E)=2$. Using Lemma $4, \operatorname{Ord}(\sigma a ; \widetilde{K})=2$. Hence, $\omega \sigma a \in B d_{1}^{\omega} \widetilde{K}$ and $\omega \sigma \in L k_{1}^{\omega}(a ; \widetilde{K})$, see Figure 6(d). Similarly, $\omega \sigma \in L k_{1}^{\omega}(b ; \widetilde{K})$. Therefore, $L k_{1}^{\omega}(a ; \widetilde{K}) \cap L k_{1}^{\omega}(b ; \widetilde{K})$ contains an edge.

In both cases the link condition (III.1) is violated because $L k_{1}^{\omega}(a b ; \widetilde{K})$ cannot contain an edge.

\subsection{2-complexes}

We now consider the analogous problem in 2D, where $K$ is a 2-complex and $E$ is a 1-complex disjoint from 
$B d_{1} K$. Vivodtzev et al. [2] describe a proof of topology preservation by claiming that $B d_{1} \widetilde{K}=E \cup B d_{1} K$. However, this is not true because $B d_{1} \widetilde{K}$ contains edges incident on $\sigma$ in addition to edges in $E \cup B d_{1} K$. For example, in Figure 5(b), the edges $\sigma a, \sigma b, \sigma c$ and $\sigma d$ are all order-1 edges.

Moreover, the arguments used in their proof infers order of an edge by considering the number of triangles shared by the edge. These arguments do not extend to the case of 3-complexes. For instance, the star of an edge in 3-complexes contains several tetrahedra but the number of tetrahedra do not directly indicate the order of the edge. Our arguments look at relationship between star of simplices in $E$ and $\widetilde{K}$ to infer the order of a simplex. This approach is more general than the earlier arguments [2] and we prove the result in the case of $2 \mathrm{D}$ meshes using arguments analogous to the 3D case.

Topology preservation of $K$ and topology preservation of $E$ while contracting edges that do not lie in $E$, can be shown using the same arguments as in the 3D case. For the sake of completeness, we describe topology preservation of $E$ when an edge from $E$ is contracted, although this is analogous to the $3 \mathrm{D}$ case.

Since $E$ is a 1-complex, $E$ has one link condition given by:

$$
L k_{0}^{\omega}(a ; E) \cap L k_{0}^{\omega}(b ; E)=\emptyset .
$$

We show that if contracting an edge $a b \in E$ violates link condition (I.0), then link conditions are violated in $\widetilde{K}$ as well. Since $E$ is a 1 -complex, only vertices can be present in $L k_{0}^{\omega}(a ; E) \cap L k_{0}^{\omega}(b ; E)$. The vertex, $v$, that violates link condition (I.0) can either be a vertex in $E$ or the dummy vertex $\omega$.

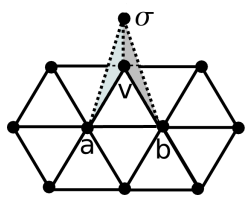

(a)

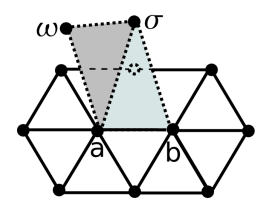

(b)
Fig. 7. (a) $\widetilde{K}$ contains $\sigma a v$ and $\sigma b v$ since cones from $\sigma$ are added to $a v$ and $b v$. (b) Cone from $\omega$ added to $\sigma a$ since $\operatorname{Ord}(\sigma a ; \widetilde{K})=1$.

Case 1. $v \in E$ :

Since $a v \in E, \sigma a v \in \widetilde{K}$. Thus, $\sigma v \in L k_{0}^{\omega}(a ; \widetilde{K})$. Similarly, $\quad \sigma v \in L_{0}^{\omega}(b ; \widetilde{K})$. Therefore, $L k_{0}^{\omega}(a ; \widetilde{K}) \cap L k_{0}^{\omega}(b ; \widetilde{K})$ contains an edge, see Figure 7(a).

Case 2. $v$ is $\omega$ :

Since cones from $\omega$ are added only to vertices of order $1, \operatorname{Ord}(a ; E)=1$. This means degree of $a$ in $E$ is either exactly one or more than two. Hence in $\widetilde{K}$, the edge $\sigma a$ is incident on either exactly one or more than two triangles. Thus $\operatorname{Ord}(\sigma a ; \widetilde{K})=1$. Hence $\omega \sigma a \in B d_{0}^{\omega} \widetilde{K}$ and $\omega \sigma \in L k_{0}^{\omega}(a ; \widetilde{K})$, see Figure $7(\mathrm{~b})$. Similarly, $\omega \sigma \in L k_{0}^{\omega}(b ; \widetilde{K})$. Therefore, $L k_{0}^{\omega}(a ; \widetilde{K}) \cap$ $L k_{0}^{\omega}(b ; \widetilde{K})$ contains an edge.

Since $\widetilde{K}$ is a 2-complex, $L k_{0}^{\omega}(a b ; \widetilde{K})$ cannot contain an edge. Hence, in both the cases $0^{t h}$ link condition is violated in $\widetilde{K}$.

\subsection{Embedded structure on boundary}

We now extend our result to include embedded structures that intersect the boundary of $K$. This assumption is a serious shortcoming of the previous result [1], [2] because embedded structures often lies on the boundary, as shown in our applications.

Lemmas 1-4 clearly show that the relation between order of a simplex in $E$ and $\widetilde{K}$ does not change whether embedded structure intersects the boundary of $K$ or not. However, Lemma 5 is not necessarily true when we remove the assumption on $E$. For example, if $E$ contains a triangle, say $a b c$, which is part of a single tetrahedron in $K$, then $\operatorname{Ord}(a b c ; K)=1$. However, the triangle $a b c \in \widetilde{K}$ is incident on two tetrahedra due to the cone from $\sigma$ and hence $\operatorname{Ord}(a b c ; \widetilde{K})=0$. Thus $B d_{1}^{\omega} K \nsubseteq B d_{1}^{\omega} \widetilde{K}$.

However, note that Lemma 5 is not necessary to show that topology of $E$ is preserved while contracting an edge. A simple solution that ensures that topology of $K$ is also preserved is to verify that link conditions of $K$ are satisfied in addition to the link conditions for $\widetilde{K}$. Although this additional validation seems to require evaluation of three new link conditions, in practice, the link conditions for $K$ and $\widetilde{K}$ can be verified in a single procedure.

\subsection{Necessity of Link Conditions}

For a manifold $M$, the link condition for an edge $a b$ is $L k(a ; M) \cap L k(b ; M)=L k(a b ; M)$. For 2-manifolds and 3-manifolds, link conditions are necessary for ensuring topology preservation [9]. The assumption of the domain being a manifold is very restrictive and does not hold in many practical situations. However, in a more general setting of non-manifolds, link conditions are not necessary for ensuring topology preservation. In this section, we show that under practically reasonable assumptions, link conditions are almost always necessary. In particular, when $K$ is a 2-manifold with or without boundary, $E$ is a 1-manifold with or without boundary, and $E \cap B d_{1} K=\emptyset$, we show that except for two special cases, the link conditions of $\widetilde{K}$ are necessary for preserving the topology of $K$ and $E$.

Since necessity of link conditions for topology preserving simplification of meshes with embedded structures were not studied earlier, it was not clear if such simplifications were too conservative and disallowed edge contractions that preserved the topology but violated link conditions. Our result shows that 2D mesh simplification with embedded structures is nearly optimal using link conditions, in the sense that most of the edge 
contractions that we disallow do in fact modify topology. Section 7.2 describes an example of link condition violation that results in modification of the topology of the embedded structure. We describe the proof of necessity of link conditions in Appendix B.

\section{IMPLEMENTATION}

Our implementation of simplification of 3D meshes with embedded structures essentially contracts edges that satisfy link conditions. The input mesh represents a 3-manifold with or without boundary. The simplification proceeds until the number of vertices in the mesh reaches a user-specified threshold, $v$, or until no edge can be contracted without violating topology, whichever happens earlier.

\subsection{Data structure and algorithm}

We represent the input mesh using the triangle-edge data structure [14]. Each triangle has a flag to identify if the triangle belongs to $E$. We use quadric error metric [6] to handle 3D meshes with a scalar field and improve the quality of the mesh [8]. In order to ensure that the geometry of the embedded structure is minimally affected, triangles in the embedded structure are treated similar to boundary triangles i.e. edges that are incident on the embedded structure are penalized with a higher weight. Edges are selected from a priority queue in the order of increasing cost. Function SIMPLIFY describes the high level algorithm. Implementation details, except for the evaluation of order of simplices in $\widetilde{K}$, can be found in earlier work [6], [8].

$\operatorname{SIMPLIFY}(K)$

Initialise priority queue $Q$ with edges in $K$

while(\# of vertices $>v$ and Q.notempty ()) do

$a b=Q \cdot p o p()$

if(Link Conditions for $K$ and $\widetilde{K}$ are satisfied)

Contract $a b$ and update $K$ and $Q$

endif

endwhile

\subsection{Computing the order of simplices in $\widetilde{K}$}

Simplices that are not part of $E$ have the same order in $K$ and $\widetilde{K}$. Hence we consider only simplices whose order is different in $K$ and $\widetilde{K}$ in this section. We describe in detail the computation of order when the embedded structure is a 2-manifold with or without boundary. For non-manifold embedded structures, the order is evaluated on a case by case basis.

Tetrahedra: All tetrahedra incident on $\sigma$ have order 0 .

Triangles: A triangle $a b c \in E \cap B d_{1} K$ is incident on exactly two tetrahedra, one in $K$ and the other a cone from $\sigma$, and hence $\operatorname{Ord}(a b c ; \widetilde{K})$ is 0 . If $a b c$ lies in the interior of $K$ then $a b c$ is incident on three tetrahedra and hence has order 1 . The triangle could also be a cone

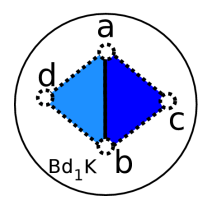

(a)

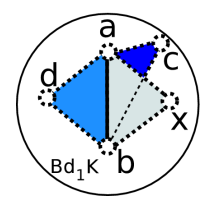

(b)

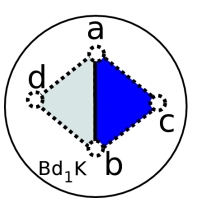

(c)

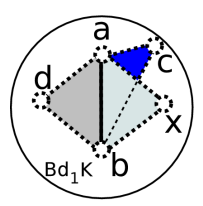

(d)
Fig. 8. (a) $S t(a b ; E)$ contains triangles $a b c$ and $a b d$, both lying in $B d_{1} K$. (b) $S t(a b ; E)$ contains triangle $a b d$ on $B d_{1} K$ and $a b c$ in the interior of $K$. (c) $S t(a b ; E)$ contains exactly one triangle $a b c$ lying in $B d_{1} K$. (d) $S t(a b ; E)$ contains exactly one triangle $a b c$ lying in the interior of $K$.

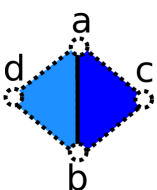

(a)

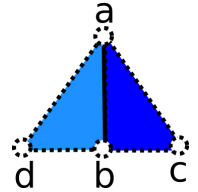

(b)

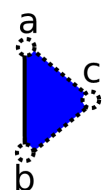

(c)

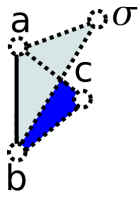

(d)
Fig. 9. (a) $S t(a b ; E)$ contains exactly two triangles $a b c$ and $a b d$ in the interior of $K$. (b) Isomorphic subdivision of star of $a c d$. (c) $S t(a b ; E)$ contains exactly one triangle $a b c$ in the interior of $K$. (d) $\sigma a b$ is incident on one tetrahedron, whereas $a b c$ is incident on three tetrahedra.

from $\sigma$, namely $\sigma a b$. The triangle $\sigma a b$ is incident on two tetrahedra if $S t(a b ; E)$ has two triangles. In this case, $\operatorname{Ord}(\sigma a b ; \widetilde{K})$ is 0 . If $S t(a b ; E)$ has exactly one triangle then $\operatorname{Ord}(\sigma a b ; \widetilde{K})$ is 1 .

Edges: For an edge $\sigma a, \operatorname{Ord}(\sigma a ; \widetilde{K})=\operatorname{Ord}(a ; E)$. For edges in $E$, we consider the following two cases.

Case 1. Edge $a b \in E$ lies on $B d_{1} K$ :

If $a b$ is incident on exactly two triangles $a b c$ and $a b d$ in $E$ and if both $a b c$ and $a b d$ are on $B d_{1} K$ as shown in Figure $8(\mathrm{a})$, then $\operatorname{Ord}(a b ; \widetilde{K})$ is 0 . This is because the half sphere $S t(a b ; K)$ grows to become a sphere in $\widetilde{K}$ after adding the cones from $\sigma$. If, at least one triangle, say $a b c$ is in the interior of $K$, as shown in Figure $8(\mathrm{~b})$ then $\operatorname{Ord}(a b ; \widetilde{K})$ is 2 . This is because $B d_{1} K$ is a 2 manifold and hence $a b$ is incident on a triangle $a b x$ on $B d_{1} K$. Triangle $a b x$ is incident on only one tetrahedron and $a b c$ on three tetrahedra. Subdividing the star of a triangle cannot create two triangles, one of which is incident on exactly one tetrahedron and the other on three tetrahedra. If $a b$ is incident on only one triangle $a b c \in E$ and $a b c$ lies on $B d_{1} K$, as shown in Figure $8(\mathrm{c})$, then $\operatorname{Ord}(a b ; \widetilde{K})=1$, since $\operatorname{St}(a b ; \widetilde{K})$ is a half sphere. If $a b c$ lies in the interior of $K$, as shown in Figure $8(\mathrm{~d})$, then $\operatorname{Ord}(a b ; \widetilde{K})=2$, due to triangles $a b x$ and $a b c$ incident on one and three tetrahedra respectively, as before.

Case 2. Edge $a b \in E$ lies outside $B d_{1} K$ :

If $S t(a b ; E)$ has exactly two triangles, then 


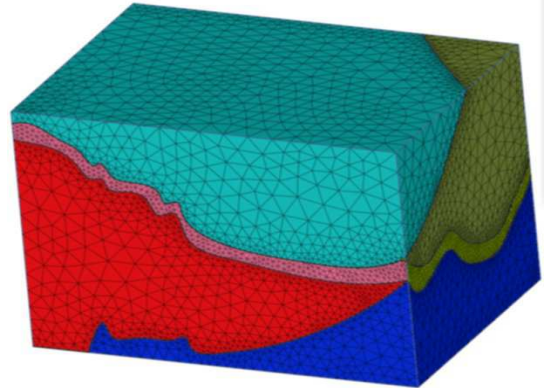

(a)

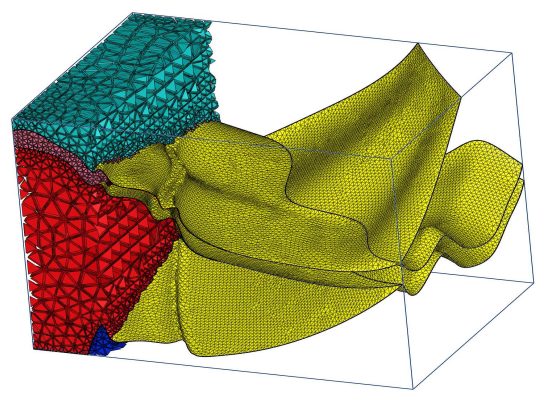

(d)

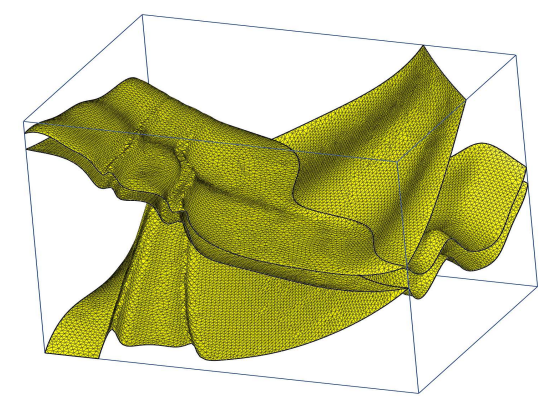

(g)

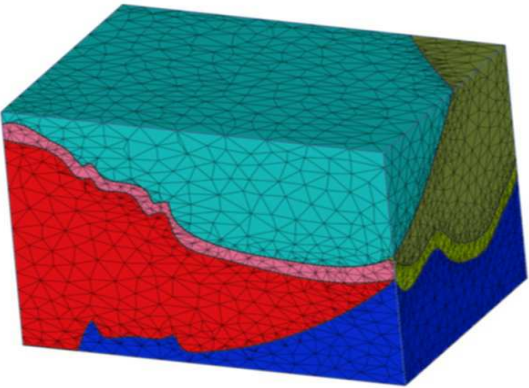

(b)

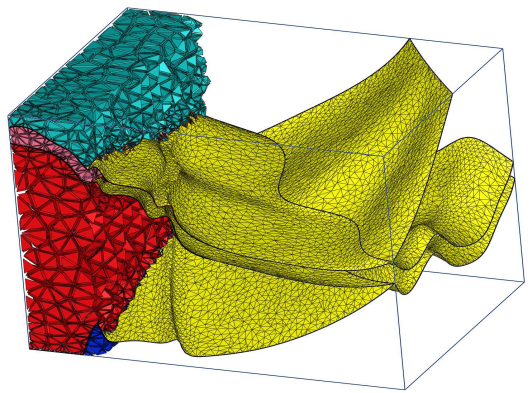

(e)

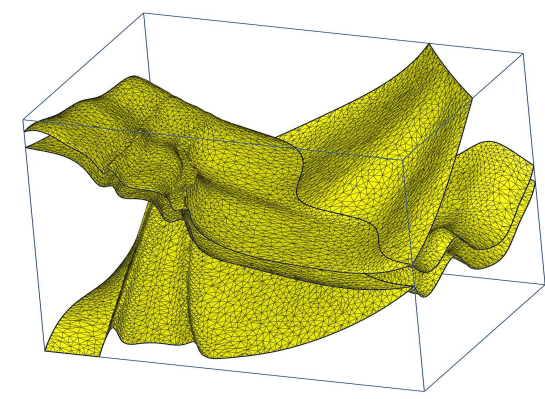

(h)

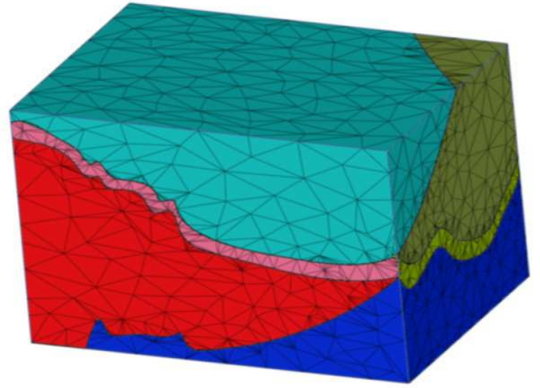

(c)

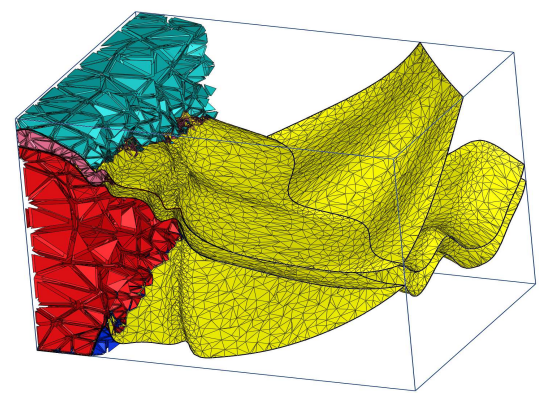

(f)

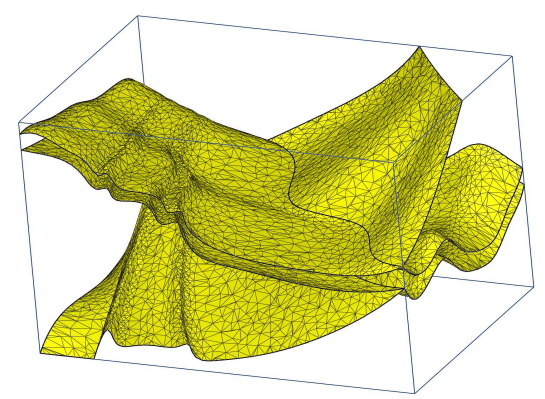

(i)

Fig. 10. Tetrahedral mesh modeling different soil types at $100 \%, 30 \%$, and $10 \%$ (from left to right) of original data. First row shows the boundary of the mesh. Second row shows a cutaway view of the mesh interior with tetrahedra on the left and the embedded surface in yellow. Third row shows the embedded surface.

$\operatorname{Ord}(a b ; \widetilde{K})=1$, because $\operatorname{St}(a b ; \widetilde{K})$ has isomorphic subdivision with star of a triangle acd $\in E$ in the interior of $K$, as shown in Figure 9(a), 9(b).

If $S t(a b ; E)$ has only one triangle $a b c$, as shown in Figure 9(c) then $\operatorname{Ord}(a b ; \widetilde{K})$ is 2 since $\sigma a b$ is incident on exactly one tetrahedron, while $a b c$ is incident on three tetrahedra, as shown in Figure 9(d).

Vertices: For a vertex $a \notin B d_{1} K, \operatorname{Ord}(a ; \widetilde{K})=$ $\operatorname{Ord}(a ; E)+1$, using isomorphic subdivisions of $\operatorname{St}(a b ; E)$ described in Figure 9(a) and Figure 9(c).

For a vertex $a \in B d_{1} K$, if $S t(a ; E) \subset B d_{1} K$, then $\operatorname{Ord}(a ; \widetilde{K})=\operatorname{Ord}(a ; E)$, using the subdivision of $S t(a b ; K)$, described in Figure 8(a) and Figure 8(c). If $\operatorname{St}(a ; E)$ is a disk such that a half disk lies on $B d_{1} K$ and the remaining half disk lies in the interior of $K$, then $\operatorname{Ord}(a ; \widetilde{K})$ is 2, using the subdivision of $\operatorname{St}(a b ; K)$, described in Figure $8(\mathrm{~b})$. If $S t(a ; E)$ is a half disk that lies in the interior of $K$, then $\operatorname{Crd}(a ; \widetilde{K})$ is 2, using the subdivision of $S t(a b ; K)$, described in Figure 8(d). Although it is possible to analyse the remaining cases when $a$ lies on $B d_{1} K$ and its star lies partially in the interior of $K$, for ease of computation and bookkeeping involved during implementation, we consider such a vertex to have order 3. Overestimating the order of the vertex assures that the topology is preserved at the cost of preventing a few legal edge contractions.

\section{Applications}

\subsection{Study of Seismic Activity}

Geophysicists model the earth at different scales in space and time in order to study different phenomena including the convection in the terrestrial crust that influences tectonic plates dynamics, the convection in the outer core 
region for the generation of earth's magnetic field [20], or at a smaller scale, the propagation of seismic waves in a particular site of earth's surface [21].

All these phenomena share in common the fact that one or more regions of homogeneous behaviour are separated by strong discontinuities that play a crucial role in the physical process. For example, in the generation of earth's magnetic field, the Gutenberg and the Lehmann discontinuities respectively separating the outer core region from the mantel above and from the inner core below are the key to the simulation of thermal convection. In the simulation of seismic waves for a specific site of earth's surface, the clear separation between different geological formation and soil types, as well as the precise modeling of faults are crucial. For example, solid rocks tend to transmit seismic waves without transformation while sandy and clay soils will amplify them.

Figure 10 shows a tetrahedral mesh modeling the different soil types and faults in a local site. Embedded structures in the mesh can be used to represent the faults and the interfaces between soil types. These embedded structures, shown in yellow, form a non-manifold surface with non-manifold edges at the intersection between interfaces. Vertices of order 3 are obtained whenever these non-manifold edges reach the boundary of the mesh. Our simplification process preserves the complex non-manifold topology of the embedded structures even at a very coarse scale.

\subsection{Isosurface Topology Preservation}

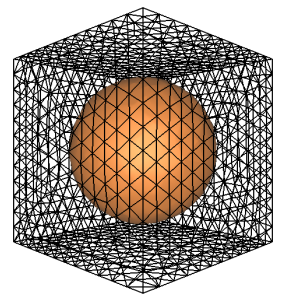

(a)

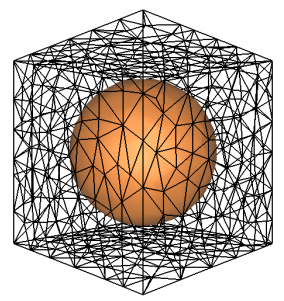

(c)

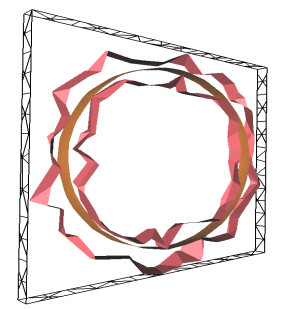

(b)

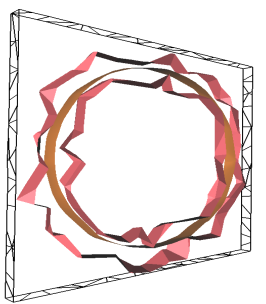

(d)
Fig. 11. (a) A cube along with an extracted isosurface shown in brown. (b) A cut section of the isosurface sandwiched between inner and outer envelopes shown in pink. (c) The simplified mesh and the extracted isosurface. (d) The cut section after simplification.

Topology of isosurfaces gives insights about important features of the underlying volumetric data. The topology

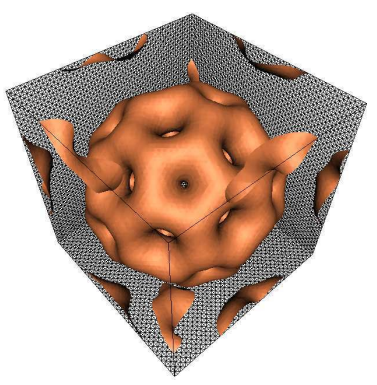

(a)

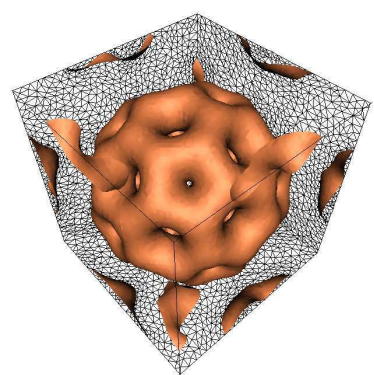

(b)

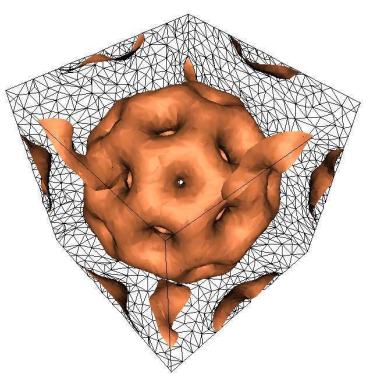

(c)

Fig. 12. Isosurface topology preserving volume simplification. (a), (b), and (c) show isosurface at $100 \%, 30 \%$, and $10 \%$ respectively of original data.

of isosurfaces may be important in medical applications like cortex labeling, organ template fitting, etc. In CAD modeling, features like tunnels and holes are used in identifying important characteristics of the model.

Our simplification technique can be applied to preserve topology of specific isosurfaces in the case of tetrahedral meshes. Triangles that constitute the isosurface need not be part of the input mesh. So, we consider an outer and inner envelope of the isosurface such that triangles in the envelope are faces of tetrahedra in the input mesh, see Figure 11. The envelope thus identifies an isovolume that contains the isosurface. We treat the inner and outer envelopes as embedded structures while simplifying the volume. Since the topology of the envelope is preserved, the topology of the isovolume enclosed by the two envelopes is also preserved. Hence the topology of the original isosurface is preserved. It may happen that the simplified envelopes are not the envelopes of the isosurfaces computed on the simplified meshes. However, we can prevent this artifact by choosing the simplified scalar field values to lie outside that of the envelope surfaces.

Figure 12 shows isosurface extracted from the original and simplified Bucky Ball dataset. The isosurface in Figure 12(b) is very similar to the original isosurface, however changes can be noticed in Figure 12(c). This happens because the edges incident on the envelopes have a higher cost and hence the volume between the envelopes change only after $70 \%$ simplification is done. 


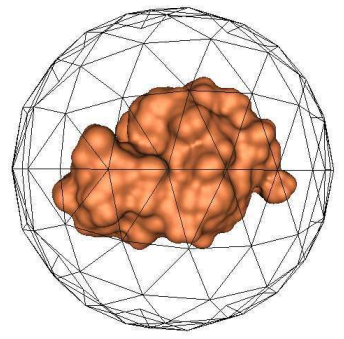

(a)

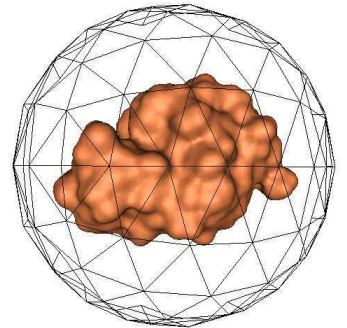

(b)

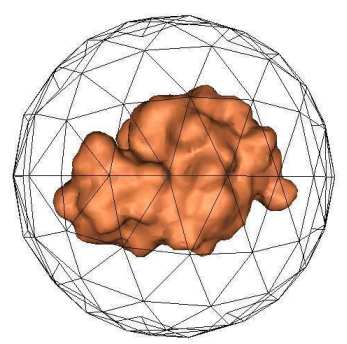

(c)
Fig. 13. Molecular surface simplification. The mesh representing the volume occupied by the protein molecule (PDB ID: 193L) is simplified while preserving the topology of the molecular surface. (a), (b), and (c) show molecular surface at $100 \%, 30 \%$, and $10 \%$ respectively of original data.

\subsection{Molecular Surface Topology Preservation}

Modeling of molecular surfaces of proteins is useful in applications like biomolecular recognition, study of drug binding cavities, etc. Preserving the topology of the molecular surface is important in studying the properties of the molecule. For example, the stability of a protein depends on the number and size of voids [18].

A molecule in solution is represented by a volume mesh. We simplify the mesh while preserving the topology of the molecular skin surface [19] , which is specified as an embedded structure. Figure 13 shows a molecular surface embedded within a tetrahedral mesh having a spherical boundary. The molecular surface is very dense in the sense that many of the vertices of the mesh lie on the molecular surface. So, most of the edges selected for contraction lie on the molecular surface. This results in the spherical boundary remaining unchanged even after removing $90 \%$ of the vertices. Our technique preserves the topology of the molecular surface even though the simplification is essentially restricted to the molecular surface.

\section{EXPERIMENTS}

We simplify four datasets and measure the root mean square error, the time taken, and the standard deviation of dihedral, solid, and face angles during simplification. Three datasets from the AIM@SHAPE repository, (BUCKY BALL, PlASMA64, and LIQUID OXYGEN POST) have a scalar field associated with them. In each case,
TABLE 1

Results of simplification of three isosurface and a molecular surface dataset. The time taken is measured on a $2 \mathrm{GHz}$ Intel Xeon CPU.

\begin{tabular}{|l||r|r|r|r|}
\hline Dataset & $\%$ & \#vert & rms & time (sec) \\
\hline & 100 & 262144 & 0 & 0 \\
Bucky Ball & 50 & 131072 & 0.024 & 34 \\
& 20 & 52428 & 0.035 & 60 \\
10 & 26214 & 0.037 & 71 \\
& 5 & 13107 & 0.041 & 78 \\
\hline \multirow{5}{*}{ Plasma64 } & 100 & 274625 & 0 & 0 \\
& 50 & 137312 & 0.032 & 35 \\
& 20 & 54925 & 0.035 & 62 \\
& 10 & 27462 & 0.036 & 72 \\
& 5 & 13731 & 0.037 & 78 \\
\hline \multirow{5}{*}{ Liquid Oxygen Post } & 100 & 108300 & 0 & 0 \\
& 50 & 54150 & 0.051 & 17 \\
& 20 & 21660 & 0.084 & 27 \\
& 10 & 10830 & 0.095 & 30 \\
& 5 & 5415 & 0.105 & 33 \\
\hline & 100 & 34920 & 0 & 0 \\
& 50 & 17460 & 0.008 & 6 \\
& 20 & 6984 & 0.021 & 11 \\
& 10 & 3492 & 0.047 & 13 \\
& 5 & 1746 & 0.127 & 14 \\
\hline
\end{tabular}

we are interested in preserving the topology of one or more isosurfaces extracted from the data. For each vertex of the original mesh, the corresponding location in the simplified mesh is determined by interpolating inside tetrahedra of the simplified mesh. The difference between the scalar field value at these two locations determine the root mean square error. The fourth dataset represents a molecule in solution (PDB ID: 193L), where the surface of the molecule is stored as the embedded structure, see Section 6.3. Error introduced by the simplification is measured as the rms distance between the two surfaces. The datasets are normalised so that the spatial coordinates and function values lie between 0 and 1 .

\subsection{Results}

Table 1 lists the average root mean square error introduced and the time taken during simplification of the four datasets. The average values of dihedral, solid, and face angles remain nearly constant, close to $1.22,0.53$, and 1.05 radians respectively. The standard deviation increases with simplification as shown in Figure 15.

\subsection{Topology violation without using Link Condi- tions}

The quadric error metric as described in Section 5.1 aims to preserve the geometry of the mesh and the embedded structure. We now illustrate, using an example, the importance of link conditions to ensure topology preservation.

Figure 14 shows a thin ring-like section in the embedded structure of the LIQUID OXYGEN POST dataset. The magnified view shows that the ring-like section is a 2-manifold with boundary. A simplification without checking link conditions of $\widetilde{K}$ creates a principal edge 


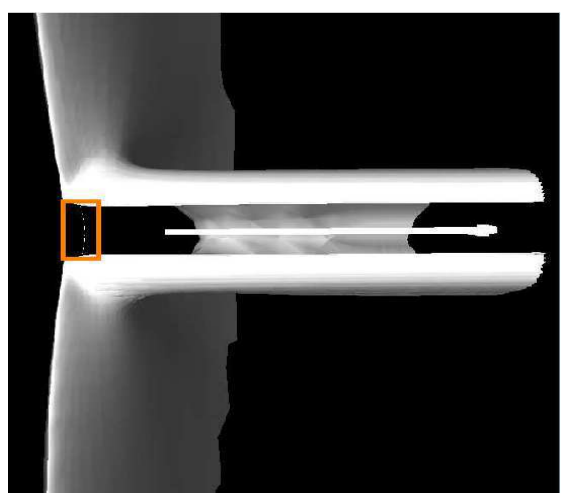

(a)

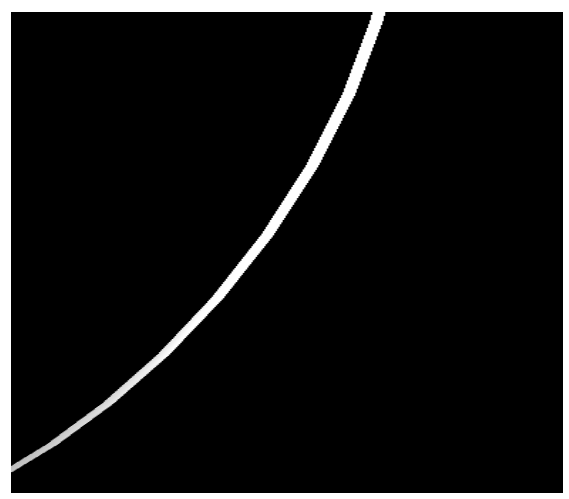

(b)

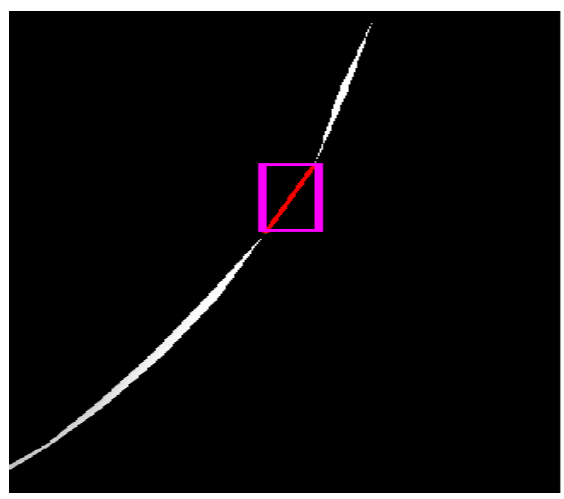

(c)

Fig. 14. Topology violation of embedded structure. (a) Embedded structure with a ring like portion. (b) Magnified view of the ring like portion which is a 2-manifold with boundary. (c) A principal edge appears (shown in red) after simplification. This indicates topology violation since the original embedded structure did not contain a principal edge.

(i.e. no cofaces). This shows violation of the topology of the embedded surface since the original embedded surface did not contain a principal edge. This example shows that a naïve simplification approach will not be suitable for applications that require guarantee about topology preservation.

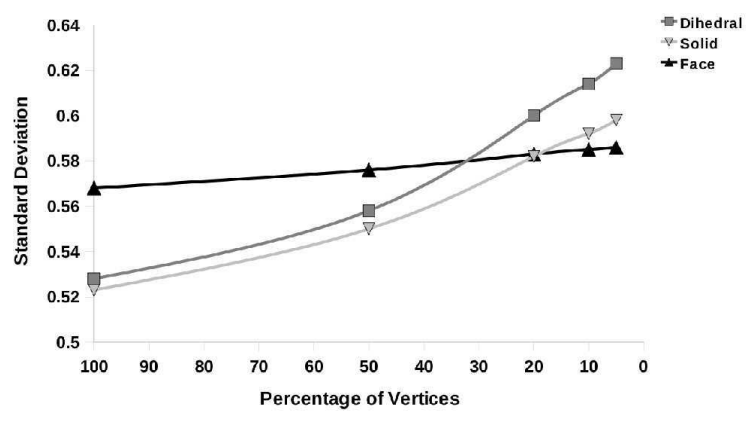

Fig. 15. Graph showing increase in standard deviation of dihedral, solid, and face angles for Liquid Oxygen Post dataset.

\section{Conclusion}

We prove theoretically the correctness of the link conditions proposed earlier [1], [2] for topology preserving simplification of meshes with embedded structures. Our approach results in a unified proof for $2 \mathrm{D}$ and $3 \mathrm{D}$ meshes. We also demonstrate usefulness of this technique in three novel applications: soil interface preservation in geophysics, isosurface topology preservation, and molecular surface topology preservation. Besides preserving the scalar field and creating good quality mesh elements, our implementation also ensures that the geometry of the embedded structure is preserved.

In this paper, we have analysed the necessity of the mesh simplification technique for 2D meshes with $1 \mathrm{D}$ embedded structures. The necessity of the technique in the case of 3D meshes needs to be analysed. Currently, we require a detailed case analysis for computing the order of simplices. We plan to explore other approaches to compute the order of a simplex. Since such techniques would measure the topological complexity of simplices, they may provide better insights in identifying important features of a mesh.

\section{APPENDIX A Proof of Lemmas}

Lemma 1. For an edge $a b \in E$, if $\operatorname{Ord}(a b ; E)=1$, then

i. $\operatorname{Ord}(\sigma a b ; \widetilde{K})=1$

ii. $\operatorname{Ord}(a b ; \widetilde{K}) \geq 1$.

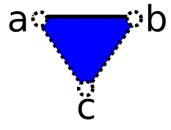

(a)

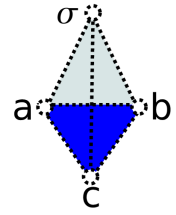

(b)

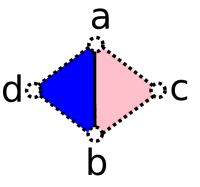

(c)

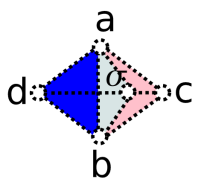

(d)
Fig. 16. (a) An order-1 edge $a b$ in $E$ and (b) the corresponding order-1 triangle $\sigma a b$ in $\widetilde{K}$. (c) An order-0 edge $a b$ in $E$ and (d) the corresponding order-0 triangle $\sigma a b$ in $\widetilde{K}$.

Proof: $\operatorname{Ord}(a b ; E)=1$. So, $\operatorname{St}(a b ; E)$ has either exactly one triangle (as shown in Figure 16(a)) or more than two triangles and hence $S t(\sigma a b ; \widetilde{K})$ has either exactly one tetrahedron (Figure 16(b)) or more than two tetrahedra. Thus $\operatorname{Ord}(\sigma a b ; \widetilde{K})=1$. Since order of a face cannot be less than its coface, $\operatorname{Ord}(a b ; \widetilde{K}) \geq 1$.

Lemma 2. For an edge $a b \in E$, if $\operatorname{Ord}(a b ; E)=0$, then $\operatorname{Ord}(\sigma a b ; \widetilde{K})=0$.

Proof: Since $\operatorname{Ord}(a b ; E)=0, a b$ is either principal or incident on exactly two triangles (Figure 16(c)). Hence in $\widetilde{K}, \sigma a b$ is principal or incident on exactly two tetrahedra (Figure 16(d)) and therefore $\operatorname{Ord}(\sigma a b ; \widetilde{K})=0$. 
Lemma 3. For a vertex $a \in E$, if $\operatorname{Ord}(a ; E) \geq 1$, then $\operatorname{Ord}(\sigma a ; \widetilde{K}) \geq 1$.

Proof: Since $\operatorname{Ord}(a ; E) \neq 0,|\operatorname{St}(a ; E)|$ is not a manifold. Hence $|S t(\sigma a ; \widetilde{K})|$ is also not a manifold. Therefore $\operatorname{Ord}(\sigma a ; \widetilde{K}) \geq 1$.

Lemma 4. For an edge $a b \in E$, if $\operatorname{Ord}(a b ; E)=1$ and $\operatorname{Ord}(a ; E)=2$, then $\operatorname{Ord}(\sigma a ; \widetilde{K})=2$.

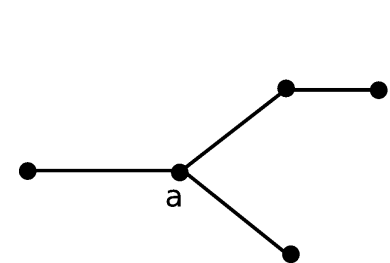

(a)

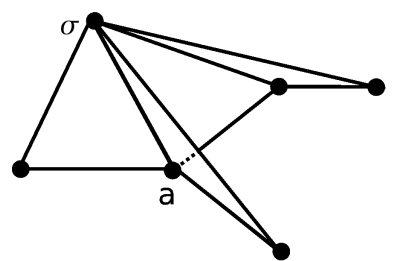

(b)
Fig. 17. (a) A 1-complex with a vertex $a$ on its 1-boundary. Note that all vertices with degree not equal to two, lie on the 1-boundary. (b) Add cones from $\sigma$ to the 1-complex. The edge $\sigma a$ is incident on three triangles and therefore lies on the 1-boundary of the 2-complex.

\section{Proof:}

Case 1. $a \in B d_{1} B d_{1} E$ :

Since all the edges in $B d_{1} E$ are order- 1 edges in $E$, in particular, the edges incident on $a$ in $B d_{1} E$ are order-1 edges in $E$. By Lemma 1, the triangles in $\widetilde{K}$ formed by adding cones from $\sigma$ to these edges have order 1 . Since $B d_{1} E$ is a 1-complex, $a$ is incident on one or more than two edges (see Figure 17(a)). Hence, the edge $\sigma a$ is incident on one or more than two triangles in $\widetilde{K}$ (see Figure 17(b)). Hence $\sigma a \in B d_{1} B d_{1} \widetilde{K}$. We know from [9] that $B d_{1} B d_{1} \widetilde{K} \subseteq B d_{2} \widetilde{K}$. Therefore $\sigma a \in B d_{2} \widetilde{K}$. Thus $\operatorname{Ord}(\sigma a ; \overline{\bar{K}})=2$.

Case 2. $a \notin B d_{1} B d_{1} E$ :

Degree of $a$ in $B d_{1} E$ is exactly 2. Besides the edge $a b$, assume that the vertex $a$ is incident on edge $a x$. Since $\operatorname{Ord}(a b ; E)=1, a b$ is incident on either exactly one or more than two triangles in $E$. Let $a b$ be incident on exactly one triangle. Since all edges incident on $a$, besides $a x$ and $a b$, have order 0 , the star of $a$ contains a half disk as shown in Figure 18. We now consider two cases based on the number of triangles incident on $a x$.

Case $2 \mathrm{a} . \quad a x$ is incident on one triangle:

In this case the sequence of triangles $a b y_{1}, a y_{1} y_{2}, \ldots, a y_{n} x$ form a half disk of triangles. If $S t(a ; E)$ is exactly a half disk, then $\operatorname{Ord}(a ; E)=1$. However, we know $\operatorname{Ord}(a ; E)=2$, hence $\operatorname{St}(a ; E)$ must contain at least one principal edge in addition to the half disk, as shown in Figure 18(a). Corresponding to each triangle, $a y_{i} y_{i+1}$ in the half disk, $\operatorname{St}(\sigma a ; \widetilde{K})$ contains the

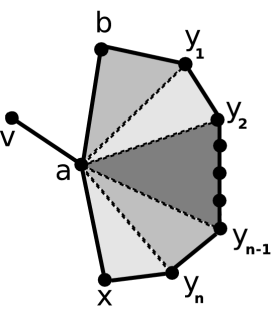

(a)

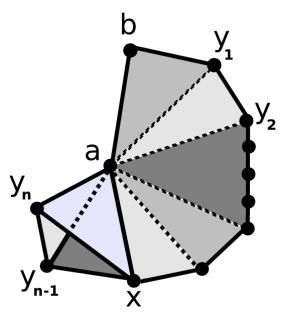

(b)
Fig. 18. (a) $\operatorname{Ord}(\sigma a ; \widetilde{K})=2$ since $S t(a ; E)$ has a half disk and a principal edge. (b) $\operatorname{Ord}(\sigma a ; \widetilde{K})=2$ since $a b$ and $a x$ are incident on one and three triangles respectively.

tetrahedron $\sigma a y_{i} y_{i+1}$ and corresponding to each principal edge $a v, S t(\sigma a ; \widetilde{K})$ contains the principle triangle $\sigma a v$. It is not possible to have isomorphic subdivisions of $\operatorname{St}(\sigma a ; \widetilde{K})$ and star of a triangle in any arbitrary complex because of the principal triangles in $\operatorname{St}(\sigma a ; \widetilde{K})$. So, $\operatorname{Ord}(\sigma a ; \widetilde{K})=2$.

Case 2b. $a x$ is incident on more than two triangles: In this case, $a b$ is incident on one triangle and $a x$ is incident on more than two triangles, as shown in Figure 18(b). Hence, $\operatorname{St}(\sigma a ; \widetilde{K})$ will contain the triangle $\sigma a b$, which is incident on only one tetrahedron, and $\sigma a x$ which is incident on more than two tetrahedra. No subdivision of star of a triangle in any arbitrary complex can contain triangles incident on one and more than two tetrahedra at the same time. Thus $\operatorname{Ord}(\sigma a ; \widetilde{K})=2$.

The above arguments extend to the case when $a b$ is incident on more than two triangles and $a b$ and $a x$ are incident on different number of triangles.

The only case remaining is when $a b$ and $a x$ do not form half disks but are incident on the same number of triangles. At least two of the triangles incident on $a b$ remain connected even when edge $a b$ is removed. This implies, at least two of the tetrahedra incident on $\sigma a b$ remain connected even when the triangle $\sigma a b$ is removed. Note that the above mentioned tetrahedra lie in $\operatorname{St}(\sigma a ; \widetilde{K})$. Now, consider a triangle in an arbitrary complex whose star contains at least three tetrahedra. One of the triangles in the subdivision of this star satisfies the property that its removal disconnects the incident tetrahedra. The existence of such a triangle implies that $S t(\sigma a ; \widetilde{K})$ and the star of a triangle cannot have isomorphic subdivisions. So, $\operatorname{Ord}(\sigma a ; \widetilde{K})=2$.

Lemma 5. $B d_{i}^{\omega} K \subseteq B d_{i}^{\omega} \widetilde{K}, \forall i \geq 0$.

Proof: Except for the simplices in $E$, all other simplices have the same star in $K$ and $\widetilde{K}$. Hence the only simplices whose order may differ between $K$ and $\widetilde{K}$ are those in $E$. For a simplex $s \in E, \operatorname{Ord}(s ; K)=0$ since $E \cap B d_{1} K=\emptyset$. Since order of a simplex is a non-negative number, 
$\operatorname{Ord}(s ; \widetilde{K}) \geq 0$. Hence $B d_{i}^{\omega} K \subseteq B d_{i}^{\omega} \widetilde{K}$.

Note that the assumption, $E \cap B d_{1} K=\emptyset$, is essentially required only for the proof of Lemma 5 .

\section{APPENDIX B}

\section{Proof of Necessity Conditions}

The link conditions for $\widetilde{K}$,

$$
\begin{aligned}
& L k_{0}^{\omega}(a ; \widetilde{K}) \cap L k_{0}^{\omega}(b ; \widetilde{K})=L k_{0}^{\omega}(a b ; \widetilde{K}), \\
& L k_{1}^{\omega}(a ; \widetilde{K}) \cap L k_{1}^{\omega}(b ; \widetilde{K})=\emptyset .
\end{aligned}
$$

are not necessary in the following special cases.

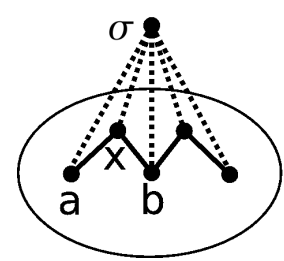

(a)

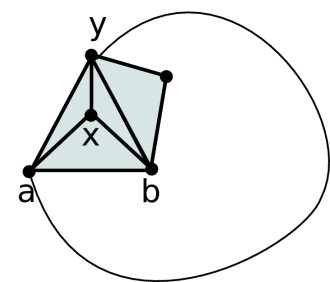

(b)
Fig. 19. Two exceptions where the edge $a b$ can be contracted without violating topology, but violates the link conditions. (a) Exception $1: a, x$, and $b$ are the first 3 vertices in $E$. (b) Exception $2: y a$ is on the boundary of $K$.

Exception 1. $L k_{0}^{\omega}(a ; \widetilde{K}) \cap L k_{0}^{\omega}(b ; \widetilde{K})$ contains an edge $\sigma x$ where $a, x$, and $b$ are three vertices of $E$ as shown in Figure 19(a). In this case, there is no topology violation since the complex before and after contraction is topologically equivalent. However, link condition (1) is violated as an edge is present in $L k_{0}^{\omega}(a ; \widetilde{K}) \cap L k_{0}^{\omega}(b ; \widetilde{K})$.

Exception 2. $L k_{0}^{\omega}(a ; \widetilde{K}) \cap L k_{0}^{\omega}(b ; \widetilde{K})$ contains an edge $x y$ such that either $y a$ or $y b$ lies on the boundary of $K$ (Figure 19(b)). If $y a$ and $y b$ were not on the boundary of $K$, then after contraction of $a b$, the edge $y c$ would have been incident on three triangles and hence caused a topology violation. However, without loss of generality, say ya is on the boundary. After contraction, $y c$ is incident on two triangles and hence there is no topology violation.

We now show that, in all other cases, whenever link conditions of $\widetilde{K}$ are violated, the topology of $K$ or $E$ changes. The link conditions of $\widetilde{K}$ could be violated in three ways:

Case 1. An edge $x y$ violates link condition (1):

Case 1a. $x y \in K$ :

Since Exception 2 is excluded, the edge $y a$ is part of two triangles in $K-$ yax and yau. Similarly, $y b$ is part of two triangles in $K-y b x$ and $y b v$ (Figure 20(a)). If $u \neq v$, after contracting $a b$ to $c$, the edge $y c$ is part of three triangles, $y c x, y c u$, and $y c v$.

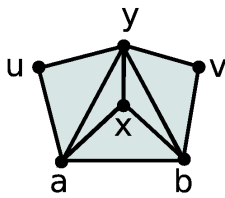

(a)

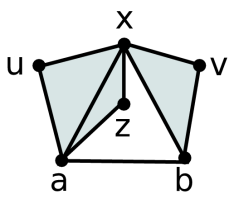

(b)
Fig. 20. (a) After contracting $a b$ to $c, y c$ becomes part of three triangles. (b) If $a b$ contracts to $c$, the edge $x c$ would be incident on three triangles.

Case 1b. $x y \notin K$ :

In $K$, no edge is part of three triangles. Thus contracting $a b$ results in change in topology of $K$. If $u=v$, then the same argument holds after replacing $x y$ with $y u$.

The only edges in $B d_{0}^{\omega} \widetilde{K}$ that are not part of $K$ are the edges incident on either $\sigma$ or $\omega$. Since all the edges in $E$ are part of three triangles in $\widetilde{K}$, the order of any edge in $E$ is 1 in $\widetilde{K}$. Hence for any simplex $s \in$ $B d_{0}^{\omega} \widetilde{K}$, if cone from $\sigma$ is added to $s$, then cone from $\omega$ is also added to $s$. So we can assume without loss of generality that $x$ is $\omega$.

If $x y$ is $\omega \sigma$, since cones from $\omega$ are added only to simplices of order 1 or higher $\operatorname{Ord}(\sigma a ; E)=\operatorname{Ord}(\sigma b ; E)=1$. This means that $\sigma a$ and $\sigma b$ are incident on a single triangle. Hence $a$ and $b$ are degree- 1 vertices in $E$. If there is no path from $a$ to $b$ in $E$, then contracting $a b$ connects two disconnected components in $E$. If the path from $a$ to $b$ in $E$ consists of only one edge, then contracting $a b$ would destroy the embedded structure. If there are more than one edge, since Exception 1 is excluded, contracting $a b$ would create a new cycle in $E$ (Figure 21(b)). Thus topology of $E$ is violated.

If $y$ is not $\sigma$, since cone from $\omega$ is added only to order- 1 edges, $a y$ and by have order 1 . Hence, $y a$ and $y b$ are either both in $E$ or $B d_{1} K$. Note that it is not possible for one edge to be in $E$ and the other to be in $B d_{1} K$ since $E \cap B d_{1} K=\emptyset$.

If $y a, y b \in E$, since Exception 1 is excluded, either $a b \in E$ or there exists edges $w a, b z \in E$. On the other hand, if $y a$, $y b \in B d_{1} K$, since $B d_{1} K$ is a 1 -manifold, either $a b \in B d_{1} K$ or there exist edges $w a$, $b z \in B d_{1} K$.

If $a b \in B d_{1} K$, then contracting $a b$ changes topology of $B d_{1} K$ because the cycle $a b-$ $b y-y a$ exists before contraction and does not exist after contraction. The same argu- 
ment holds if $a b \in E$. So, we can assume that the path $w a-a y-y b-b z$ exists in either $B d_{1} K$ or $E$ (Figure 21(a)). After contracting $a b$ to $c$, the edges $w c, y c$, and $z c$ are incident on $c$ and thus either $B d_{1} K$ or $E$ becomes a non-manifold after contraction. If $w=z$, then a cycle is destroyed.

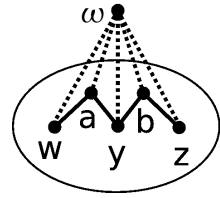

(a)

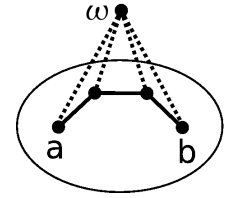

(b)
Fig. 21. (a) $B d_{1} K$ or $E$ becomes a non-manifold after contracting $a b$. (b) Contracting edge $a b$ creates a new cycle in $B d_{1} K$ or $E$.

Case 2 . Link condition (1) is violated only by vertices, say vertex $x$ :

If $x$ is not $\sigma$ or $\omega$, then edge $a x, b x$, and $a b$ exist, but the triangle $a x b$ does not exist. Since $K$ cannot have principal edges, there exists triangles $a x u$ and $b x v$. We can assume that $u \neq v$, since $L k_{0}^{\omega}(a ; \widetilde{K}) \cap L k_{0}^{\omega}(b ; \widetilde{K})$ does not contain any edges. If both $a x$ and $b x$ were part of exactly one triangle, then $L k_{0}^{\omega}(a ; \widetilde{K}) \cap L k_{0}^{\omega}(b ; \widetilde{K})$ would have contained the edge $\omega x$. Hence we can assume that $a x$ is shared by a second triangle, $a x z$ (Figure 20(b)). After contracting $a b$ to $c$, the edge $x c$ becomes part of three triangles, $x z c$, $x c u$, and $x c v$ and thus topology of $K$ changes. If $x$ is either $\sigma$ or $\omega$, then $a$ and $b$ are vertices in $E$ or $B d_{1} K$. We can assume without loss of generality that $x$ is $\omega$ since all the cones added from $\sigma$ are also added from $\omega$. If there is no path from $a$ to $b$ in link of $\omega$ then contracting $a b$ would violate topology as it would connect two components which are disconnected in $B d_{1} \widetilde{K}$. If there is a path from $a$ to $b$ in link of $\omega$, then since $L k_{0}^{\omega}(a ; \widetilde{K}) \cap L k_{0}^{\omega}(b ; \widetilde{K})$ contains no edges, the path contains at least 3 edges (Figure 21(b)). Otherwise if $a y, y b$ are the only two edges, then edge $\omega y \in L k_{0}^{\omega}(a ; \widetilde{K}) \cap L k_{0}^{\omega}(b ; \widetilde{K})$. Hence contracting $a b$ introduces a new cycle and changes topology of $E$ or $B d_{1} K$.

Case 3. Link condition (2) is violated by a vertex $x$ : If $x \in K$, then the cycle $a x, b x$, and $a b$ exists in $E \cup B d_{1} K$ and the cycle would be destroyed by contracting $a b$ thus changing the topology of $E$ or $B d_{1} K$. If $x$ is $\omega$ or $\sigma, a$ and $b$ are degree- 1 vertices in $E$. We have already considered this case earlier in Case $1 b$.

\section{ACKNOWLEDGMENTS}

We thank Dr. Fabien Vivodtzev for his valuable inputs and contributions to this paper. We thank Dr. Basudeb
Datta for discussions on the correctness of proof of topology preservation, and Dr. Philippe Gueguen for discussions on applications in geophysics. We also thank Dr. Xinwei Shi for skin meshing software and AIM@SHAPE repository for providing interesting datasets.

\section{REFERENCES}

[1] F. Vivodtzev, G.P. Bonneau, S. Hahmann, H. Hagen, "Substructure Topology Preserving Simplification of Tetrahedral Meshes", Proc. TopoInVis, 2009.

[2] F. Vivodtzev, G.P. Bonneau, P.L. Texier, "Topology Preserving Simplification of 2D Non-Manifold Meshes with Embedded Structures", The Visual Computer Journal, 21(8), pp. 679-688, 2005. Special issue of Pacific Graphics 05, Macao, China, Oct. 2005.

[3] P. Cignoni, C. Montani, Scopigno, R., "A comparison of mesh simplification algorithms" , Comput. Graph. 22(1), 37-54, 1998.

[4] M. Garland, "Multiresolution modeling: survey and future opportunities", Eurographics '99 State of the Art Reports, pp. 111-131, 1999.

[5] D.P. Luebke, "A developer's survey of polygonal simplification algorithms", IEEE Comput. Graph. Appl. 21(3), 24-35, 2001.

[6] M. Garland, P.S. Heckbert, "Surface simplification using quadric error metrics", Comput. Graph. 31, 209-216, 1997.

[7] M. Garland, P. Heckbert, "Simplifying Surfaces with Color and Texture using Quadric Error Metrics", In Proceedings of IEEE Visualization, 1998.

[8] V. Natarajan, H. Edelsbrunner, "Simplification of three-dimensional density maps", IEEE Transactions on Visualization and Computer Graphics, 10(5), 587-597, 2004.

[9] T.K. Dey, H. Edelsbrunner, S. Guha, D. V. Nekhayev, "Topology preserving edge contraction", Publ. Inst. Math. (Beograd) (N.S), 66:23-45, 1999.

[10] I. Guskov, Z. Wood, "Topological noise removal", In Proc. Graphics Interface, pages 19-26, 2001.

[11] A. Gyulassy, V. Natarajan, V. Pascucci, P.T. Bremer, B. Hamann, "Topology-based simplification for feature extraction from $3 \mathrm{~d}$ scalar fields", In Proc. IEEE Conf. Visualization, pages 535-542, 2005.

[12] A. Gyulassy, V. Natarajan, V. Pascucci, P. T. Bremer, B. Hamann, "A topological approach to simplification of three-dimensional scalar fields", IEEE Transactions on Visualization and Computer Graphics (special issue IEEE Visualization 2005), pages 474-484, 2006.

[13] P. Lindstrom, C. T. Silva, "A memory insensitive technique large model simplification", Proceedings of the conference on Visualization 01. IEEE Computer Society, pp. 121-126, 2001.

[14] E. Mucke, "Shapes and Implementations in Three Dimensional Geometry", PhD thesis, University of Illinois at UrbanaChampaign, 1993.

[15] D.W. Barnette, A.L. Edelson, "All 2-manifolds have finitely many minimal triangulations", Israel J. Math., 67(1):123-128, 1989.

[16] S.A. Lavrenchenko, "Irreducible triangulations of a torus", Ukrain. Geom. Sb., (30):52-62, ii, 1987.

[17] H.T. Vo, S.P. Callahan, P. Lindstrom, V. Pascucci, C.T. Silva, "Streaming Simplification of Tetrahedral Meshes", IEEE Transactions on Visualization and Computer Graphics, 145-155, 2007.

[18] S. Chakravarty, A. Bhinge, R. Varadarajan, "A procedure for detection and quantitation of cavity volumes in proteins: application to measure the strength of the hydrophobic driving force in protein folding", J. Biol. Chem 277:31345-31353, 2002.

[19] H.L. Cheng, X. Shi, "Quality mesh generation for molecular skin surfaces using restricted union of balls", Comput. Geom. 42(3): 196-206, 2009.

[20] G.A. Glatzmaier, P.H. Roberts, "A three-dimensional selfconsistent computer simulation of a geomagnetic field reversal," Nature, 377, 203-209, 1995.

[21] De la Puente, J., M. Dumbser, M. Kaser, H. Igel, "Discontinuous Galerkin Methods for Wave Propagation in Poroelastic Media", Geophysics, 73(5), T77-T97, 2008. 


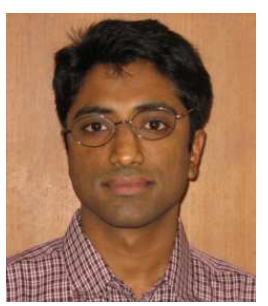

Dilip Mathew Thomas is a Ph.D. candidate in Computer Science at Indian Institute of Science, Bangalore. He received $B$.Tech. degree from National Institute of Technology, Calicut, and M.E. degree from Indian Institute of Science, both in Computer Science and Engineering. His research interests include scientific visualization and computational topology.

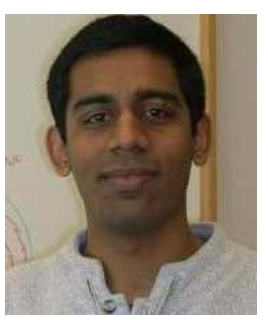

Vijay Natarajan is an assistant professor in the Department of Computer Science and Automation and the Supercomputer Education and Research Centre at the Indian Institute of Science, Bangalore. He received the Ph.D. degree in computer science from Duke University in 2004 and holds the B.E. degree in computer science and M.Sc. degree in mathematics from Birla Institute of Technology and Science, Pilani, India. His research interests include scientific tional topology, and meshing. visualization, computational geometry, computa-

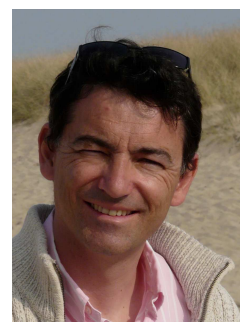

Georges-Pierre Bonneau graduated from the Ecole Normale Supérieure de Cachan, France, and received the $\mathrm{PhD}$ degree in Computer Science in 1993 from the University of Kaiserslautern, Germany. He is professor in computer science at Grenoble University, France. His research interests include multiresolution analysis, graphics applications of visual perception, scientific visualization and computer aided geometric design. He has served on the editorial board committee of IEEE Transactions on Visualization and Computer Graphics from 2002 to 2006. He co-organized two Dagstuhl seminars on scientific visualization, and has been member of international program committees of many graphics conferences. 